\title{
Emprendimiento como alternativa de inclusión laboral en personas con discapacidad en Latinoamérica: Una revisión sistemática entre los años 2010- 2020
}

\section{Entrepreneurship as an alternative for employment inclusion of people with disabilities in Latin America: A systematic review between the years 2010- 2020}

Jorge Alberto Vargas-Merino

Universidad Privada del Norte, Lima, Perú

Universidad Privada Norbert Wiener, Lima, Perú

jorgealbvarmer5@gmail.com

iD https://orcid.org/0000-0002-3084-8403

Walter Sánchez-Esli

Universidad Privada del Norte, Lima, Perú

walter.sanchez.esli@hotmail.com

https://orcid.org/0000-0001-9389-1591

Recepción: 20/07/2021 | Aceptación: 15/10/2021 | Publicación: 30/10/2021

Cómo citar (APA, séptima edición):

Vargas-Merino, J.A., y Sánchez-Esli, W. (2021). Emprendimiento como alternativa de inclusión laboral en personas con discapacidad en Latinoamérica: Una revisión sistemática entre los años 2010-2020. INNOVA Research Journal, 6(3.1), 1-18. https://doi.org/10.33890/innova.v6.n3.1.2021.1827

\section{Resumen}

En el mundo, la población con discapacidad enfrenta un gran número de retos al intentar suplir sus necesidades básicas debido a que no es insertada integralmente en el mercado laboral, principalmente cuando poseen una condición adversa significativa. Ante tal situación, el emprendimiento aparece como una alternativa y estrategia social que permite el autoempleo de dichos ciudadanos, mientras se aporta al crecimiento económico. El presente artículo tiene como objetivo analizar los estudios teóricos y empíricos sobre el emprendimiento como alternativa de inclusión laboral en las personas con discapacidad en Latinoamérica en los últimos 10 años (20102020). Este documento es una revisión sistemática de literatura científica realizada en las siguientes bases de datos: Dialnet, PROQUEST, Redalyc, Scielo y EBSCO, sumado a aportes de 
SCOPUS; se hallaron 724 artículos del tema de investigación de diversas disciplinas, posteriormente, siendo estos depurados para finalmente dar una selección de 41 artículos y 1 libro por la relevancia de sus aportes. Las conclusiones de este documento revelan que no existe una gran cantidad de estudios referentes al emprendimiento como alternativa de inclusión laboral en personas con discapacidad, estos últimos tienen poca oportunidad laboral aun cuando existen normas y leyes que traten de equiparar dicha situación, igualmente se denota una cierta falta de sensibilidad y compromiso humano con respecto a la condición laboral de las personas con discapacidad, es un aspecto social hablado, pero no practicado. Finalmente, se sugiere realizar una investigación empírica, cuantitativa, multivariada, que analice causas y efectos en temas relacionados a la inserción laboral de personas con discapacidad.

Palabras claves: emprendimiento; discapacidad; inserción laboral; empleabilidad; inclusión laboral.

\begin{abstract}
In the world, people with disabilities face a great number of challenges when trying to meet their basic needs because they are not fully inserted in the labor market, mainly when they have a significant adverse condition. In this situation, entrepreneurship appears as an alternative and social strategy that allows self-employment of these citizens, while contributing to economic growth. This article aims to analyze the theoretical and empirical studies on entrepreneurship as an alternative for labor inclusion in people with disabilities in Latin America in the last 10 years (2010-2020). This paper is a systematic review of scientific literature conducted in the following databases: Dialnet, PROQUEST, Redalyc, Scielo and EBSCO, added to SCOPUS contributions; 724 articles of the research topic from various disciplines were found, subsequently, being these debugged to finally give a selection of 41 articles and 1 book for the relevance of their contributions. The conclusions of this document reveal that there is not a large number of studies on entrepreneurship as an alternative for labor inclusion in people with disabilities, the latter have little job opportunity even when there are rules and laws that try to equalize this situation, also denotes a certain lack of sensitivity and human commitment regarding the working condition of people with disabilities, it is a social aspect spoken, but not practiced. Finally, it is suggested to carry out an empirical, quantitative, multivariate research, which analyzes causes and effects in issues related to the labor insertion of people with disabilities.
\end{abstract}

Keywords: entrepreneurship; disability; job placement; employability; labor inclusion.

\title{
Introducción
}

En el mundo, la población con discapacidad ha tenido que enfrentar muchos retos para tratar de cubrir necesidades básicas que todo ser humano debe poseer, como lo son: acceder a un sistema de prestaciones de salud, tener una vivienda adecuada, acceder a sistemas educativos y un empleo formal sin discriminación (Meléndez, 2016), existiendo políticas internacionales y nacionales que buscan cubrir dichas necesidades (Quiroz et al., 2017).

En un panorama mundial, se podría decir que el 15\% de la población presenta algún tipo de discapacidad, pero a pesar de ello, son pocos los que pueden acceder o participar de actividades que mejoren su calidad de vida logrando su bienestar integral (Galarza, 2020). Con ello, surge con 
Emprendimiento como alternativa de inclusión laboral en personas con discapacidad en Latinoamérica: Una revisión sistemática ente los años 2010-2020

fuerza el "Paradigma Social de la discapacidad", planteando que tal condición no radica en deficiencias funcionales en las personas, sino que crea barreras en la interacción entre las personas y su entorno, limitando la plena participación en diferentes ámbitos de la vida social (Vallejos, 2014).

Pico y Torres (2017) refieren que las personas con discapacidad tienen muy poca participación en el ámbito laboral, y más aún si tienen alguna discapacidad muy significativa, al ser el mercado laboral un ambiente áspero para dicha población se requiere el compromiso y la sensibilización de las autoridades competentes, empresas privadas, organizaciones civiles y la misma sociedad para buscar la inclusión laboral de la persona con discapacidad (Hernández et al., 2017). Incluso, la particularidad de la discapacidad puede desarrollar habilidades para el escenario en el que va a desempeñar su trabajo, buscando un entorno más accesible (Morales, 2015).

En el año 2015, la Organización Internacional del Trabajo (OIT) ampara una formación compartida de la persona con discapacidad y demás trabajadores en igualdad de condición y remuneración buscando la equidad de oportunidades y derechos laborales (Sánchez et al., 2019). Por otro lado, la Convención Internacional de los derechos de las Persona con Discapacidad, impulsa la inclusión laboral de la persona con discapacidad, buscando una perspectiva más favorable y de oportunidades laborales (Moreno et al., 2013); Sin embargo, a pesar de tales esfuerzos, la inclusión de dicha población aún tiene fallas que tiene que corregirse con el tiempo (Pico y Torres, 2017). Una de las dificultades para la empleabilidad e inserción laboral son las barreras culturales que generan una gran problemática social (Sempertegui, 2015).

La inclusión laboral se comprende como la inserción total e incondicional de un grupo de personas a una determinada área laboral sin importar cualquier condición adversa, esta busca beneficiar a todas las personas por igual, entendiendo que la sociedad debe contemplar también las necesidades de la población con discapacidad (Meléndez, 2016), velando por sus derechos (Reyes, 2015). Aún queda un largo camino para que las personas con discapacidad se incorporen como grupo de interés en dichas organizaciones y de esta manera puedan acceder, permanecer y ascender a un trabajo en el mercado laboral (Marrugo y Bernal, 2017).

Lo cierto es que la desigualdad persiste aún en la esfera productiva, aunque se tenga grandes avances en el reconocimiento de los derechos de las personas con discapacidad (Mercado et al., 2013). Ante esto, corresponde al estado poner mecanismos de control para brindar dispositivos que sean prácticos y utilizables por las empresas para fomentar la contratación e inclusión en el trabajo (Velarde et al., 2018). También, es necesario recalcar que la población con discapacidad presenta dificultades para mantener su puesto de trabajo, es por eso necesario crear mecanismos para fortalecer su estabilidad laboral reconociendo sus derechos (Manuera, 2015).

En los últimos diez años, el emprendimiento se ha convertido en una estrategia social y de conocimiento que permiten desarrollar habilidades para realizar proyectos empresariales buscando el autoempleo, mejorando así la calidad de vida de las personas (Vicente et al., 2018). Constituye también una alternativa que agrega valor a los objetivos económicos y sociales en el desarrollo de las naciones, así que el emprendedor se convierte en agente activo que aporta ideas creativas e innovadoras, como respuestas a las necesidades del entorno (Paz et al., 2020). 
En la actualidad, se conocen como sociedades más desarrolladas a aquellas que cuentan con un sistema de emprendimiento innovador, buscando no solo una economía más competitiva, también una sociedad más justa, solidaria y sostenible (Julia et al., 2020), es así que el emprendimiento tiene un enfoque social a partir de un modelo de negocio inclusivo (Vásquez, 2018), formando parte de una solución a la problemática social como es la pobreza y el desempleo, poco acceso a la educación y servicios de salud (Valdés y Saavedra, 2019).

Un emprendedor puede crear una empresa por necesidad o por oportunidad utilizando el ecosistema emprendedor, que une las condiciones económicas y sociales, siendo este el contexto donde el empresario puede realizar sus actividades económicas (Parga y Silva, 2020). Por consiguiente, la pregunta que surge es ¿Qué se conoce del emprendimiento como alternativa de inclusión laboral en las personas con discapacidad en los últimos 10 años?

El emprendimiento ha tomado la forma de un "motor de desarrollo económico y social", generando un cambio en la vida de las personas y nuestra sociedad, una cultura emprendedora que busca a su vez un emprendimiento social (Morales et al., 2019). Y la principal motivación que lleva a una persona con discapacidad buscar ser un emprendedor son sus creencias religiosas, perseverancia, redes sociales y soporte familiar (Núñez et al., 2019). Ante ello, surge el objetivo principal de la presente investigación que es analizar los estudios teóricos y empíricos sobre el emprendimiento como alternativa de inclusión laboral en la persona con discapacidad en los últimos 10 años.

El emprendimiento surge como una alternativa para mejorar objetivos económicos y sociales que necesita un país. El sector empresarial es protagonista del mercado laboral y un actor fundamental en la realización de una verdadera inclusión laboral de las personas con discapacidad (Pico y Torres, 2017). Se utiliza como parte de una dimensión estratégica estimular a los agentes responsables de las empresas privadas iniciar experiencias inclusivas dentro de sus organizaciones (Ramírez y Ramírez, 2015), entonces técnicamente se torna importante desarrollar un modelo de gestión inclusiva, dinámico, colaborador que sume fuerzas para cumplir los requerimientos legales y sociales para las personas con discapacidad (Lucero y Romero, 2016).

El emprendimiento empresarial se presenta como una corriente de vanguardia en los estudios y análisis contemporáneos alrededor de metas de innovación, crecimiento económico y desarrollo social (Tello, 2016). Reconociendo entonces que la población con discapacidad requiere de una atención para brindarles oportunidades de crecimiento e independencia económica. En lo que respecta al ámbito laboral, la persona con discapacidad tiene el derecho de trabajar en igualdad de condiciones respaldándose en normativas y leyes supranacionales que deben ser respetados.

\section{Metodología}

Este documento es una revisión sistemática, entiéndase esta como un resumen claro y estructurado de una información con el objetivo de responder una pregunta, para ello se buscó múltiples artículos y fuentes de información, es considerado dentro de la evidencia con el más alto nivel de jerarquía (Moreno et al., 2018). Esta se puede comprender como un estudio "integrativo, 
observacional, retrospectivo, secundario", que permite responder la misma pregunta, pero logrando una integración de estudios referentes al tema (Beltrán, 2005).

La metodología de la investigación es "la disciplina que se encarga del estudio crítico de los procedimientos, y medios aplicados por los seres humanos, que permiten alcanzar y crear el conocimiento en el campo de la investigación científica. Es necesario trabajar de manera ordenada, organizada y sistemática, depurando temas que no sean claros y permitan restructurar los indicadores si fuese necesario (Gómez, 2012).

De acuerdo con su naturaleza de objetivos, la presente investigación tiene como característica el ser descriptiva dado que se refleja los componentes principales como son las características socio laborales de este grupo vulnerable, al mismo tiempo, permite identificar las necesidades y dificultades presentadas para el emprendimiento y la inserción laboral; ya que las condiciones existentes aún no son determinantes.

En consecuencia, el presente estudio de revisión sistemática de la literatura científica permitirá conocer el "emprendimiento como una alternativa de inclusión laboral"; para ello se presenta de forma sistemática la estrategia de búsqueda a partir de definición de la pregunta ¿Qué se conoce del emprendimiento como alternativa de inclusión laboral en las personas con discapacidad en Latinoamérica en los últimos 10 años (2010-2020)? La fuente de investigación que se utilizaron para la presente revisión sistemática de la literatura se realizó en cinco plataformas de bases de datos como son: Scielo, Redalyc, ProQuest, EBSCO y Dialnet, buscando publicaciones comprendidas entre los años 2010 y 2020 (se priorizaron estas bases de datos debido a la accesibilidad y disponibilidad al momento de la recolección de artículos). A pesar de ello, se han considerado artículos de SCOPUS por su relevancia y alto impacto.

\section{Figura 1}

\section{Fuente de Información}

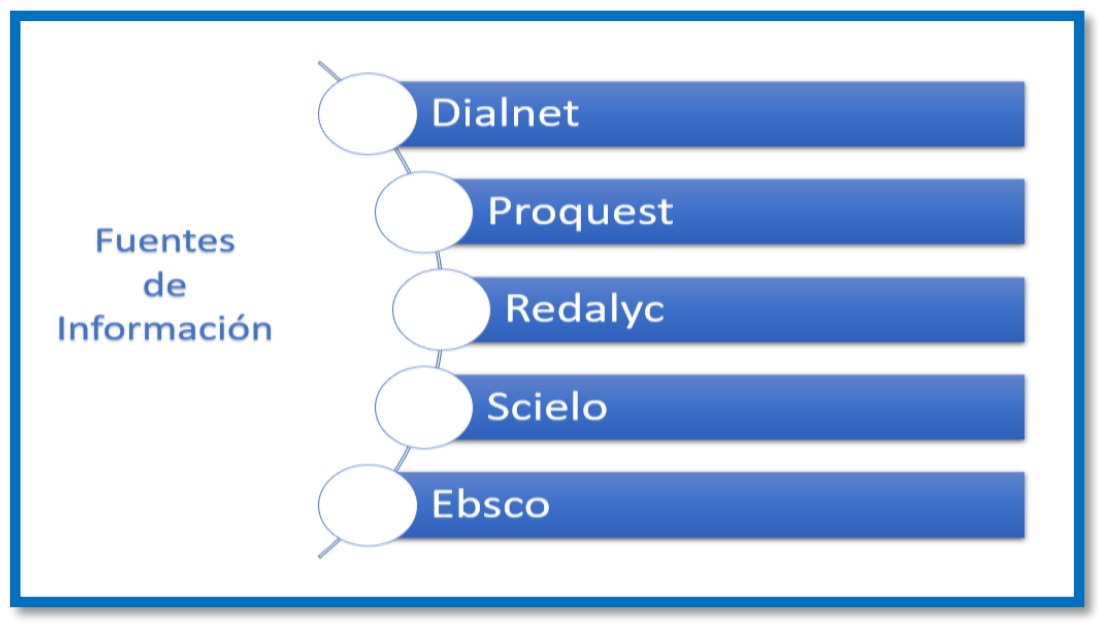

Fuente: Elaboración propia

Para el desarrollo de la presente revisión sistemática se utilizaron las diferentes fuentes de investigación, extrayendo sus diferentes artículos científicos. 
En la indagación de la revisión científica se pudo encontrar un total de 724 artículos; dichos artículos fueros seleccionados teniendo en cuenta ciertos criterios como son: ubicación geográfica, publicaciones arbitrarias, Artículos de los últimos 10 años. por las disciplinas de Administración, Salud, Multidisciplinarias (ciencias sociales) y política; recolectados en las plataformas antes mencionadas; de los cuales 80 publicaciones de Redalyc, 139 de Scielo, 292 de Proquest, 81 de Ebsco y 132 publicaciones de Dialnet, estos pasaron por criterios de inclusión y exclusión para poder discriminar los artículos relacionados con los subtemas planteados.

Se estableció como palabras claves de búsqueda: "Emprendimiento y Conceptualizaciones del emprendimiento", "Emprendimiento y tipos de emprendimiento", "Emprendimiento y perfiles del emprendedor", "Emprendimiento y estrategias para logar emprendimientos exitosos", "Inclusión laboral y discapacidad", "Inclusión laboral y política social", "Inclusión laboral y empleabilidad, "Inclusión laboral y desarrollo profesional", "Inclusión laboral y microemprendimiento", "Emprendimiento y microemprendimiento", "Empleabilidad" y "Emprendimiento". Aplicando filtros de búsqueda como el año de publicación, el idioma y País, facilitando así en tener una base datos más ajustada a la investigación.

Para la realización de la presente investigación sistemática se determinaron los criterios de inclusión se utilizaron los relacionados con los subtemas planteados (Emprendimiento, inclusión laboral, Empleabilidad), artículos originales, el año de publicación del 2010 al 2020, escritos en español o inglés y los que estén relacionados con el tema de investigación directamente, y que sean de países de Latinoamérica, por ser países en donde las políticas para las personas con discapacidad están emergiendo y necesitan una revisión de su cumplimiento. Respecto a los criterios de exclusión, se tomaron en cuenta los artículos que no se relacionaban con los subtemas planteados, temas no ubicados dentro del interés de estudio y temas que fueron publicados por duplicado que se observaron al momento de revisar los resúmenes respectivos y el título del artículo.

\section{Tabla 1}

Criterios de inclusión y exclusión de documentos

\begin{tabular}{lllll}
\hline $\begin{array}{l}\text { Tipo de } \\
\text { Documento }\end{array}$ & Periodo & Idioma & Estatus & Tipo \\
\hline & & & & Revistas \\
Artículos & Investigado del & \multirow{2}{*}{ Español } & Publicado en & Científicas, \\
Científicos & 2010 al 2020 & & Acreditado & Artículos de \\
& & & & Investigación, \\
& & & Publicaciones \\
\hline
\end{tabular}

Fuente: Elaboración propia

En la presente tabla podemos apreciar los criterios utilizados para la inclusión y exclusión de los artículos científicos, tomando en cuenta periodo, idioma, estatus y tipo.

Para una mejor sistematización de información se registró todos los artículos encontrados en una tabla de Excel, estableciendo criterios para seleccionar los artículos que ayuden en la 
Emprendimiento como alternativa de inclusión laboral en personas con discapacidad en Latinoamérica: Una revisión sistemática ente los años 2010-2020

revisión sistemática de la literatura sobre Emprendimiento como alternativa de Inclusión Laboral en Personas con Discapacidad en los últimos 10 años.

\section{Tabla 2}

Criterios para la codificación de datos

\section{Criterios para seleccionar los artículos para la codificación de datos}

\begin{tabular}{ll} 
Año de Publicación & Desde el año 2010 hasta inicios de 2020 \\
Tipo de Publicación & Publicaciones Académicas \\
& Revistas científicas \\
\hline Temas & Emprendimiento \\
& Conceptualizaciones de emprendimiento \\
& Tipos de emprendimiento \\
& Perfiles del emprendedor \\
& Inclusión laboral \\
& Discapacidad \\
& Política social \\
& Empleabilidad \\
& Ocupabilidad7 \\
& Desarrollo profesional \\
& Argentina \\
& Chile \\
País & Colombia \\
& Ecuador \\
& Paraguay \\
Perú \\
Venezuela \\
México \\
España \\
\hline Edioma
\end{tabular}

Fuente: Elaboración propia

En la presente tabla se puede apreciar la codificación de datos de los artículos científicos, tomando en cuenta el año, tipo de publicación, tema, país e idioma.

La metodología de búsqueda utilizada amplió más los criterios del tema de interés y respondió a la pregunta planteada, generando más herramientas de estudio para sustentar dicho trabajo. La estrategia de búsqueda de información académica propuesta, permite recuperar artículos que son pertinentes para el investigador y profesionales en su práctica diaria.

\section{Resultados}

De acuerdo con lo analizado en la revisión sistemática de la literatura, se obtuvieron como resultados 724 artículos científicos que tratan sobre Emprendimiento e Inclusión Laboral para personas con discapacidad, de las siguientes bases de datos, se obtuvo los artículos 
correspondientes: DIALNET 132, PROQUEST 292, REDALYC 80, SCIELO 139, EBSCO 81, teniendo como artículos totales excluidos 693 que no aportaban a nuestro tema de investigación. Por lo tanto, se seleccionaron en 39 artículos científicos que cumplieron los criterios de inclusión para la realización de la presente revisión sistemática de la literatura.

\section{Figura 2}

Proceso de selección de artículos

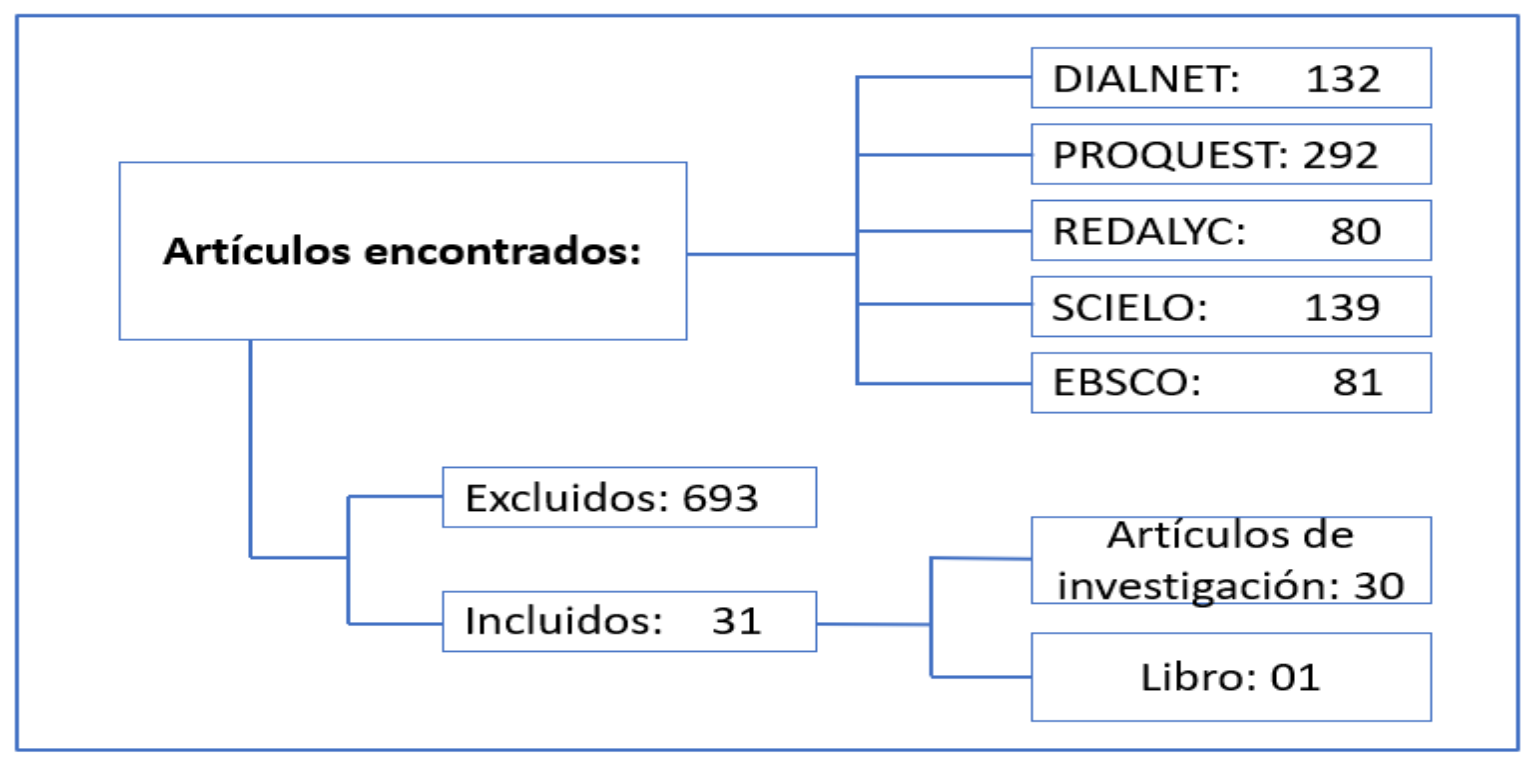

Fuente: Elaboración Propia

\section{Figura 3}

Información recolectada en porcentaje según base de datos

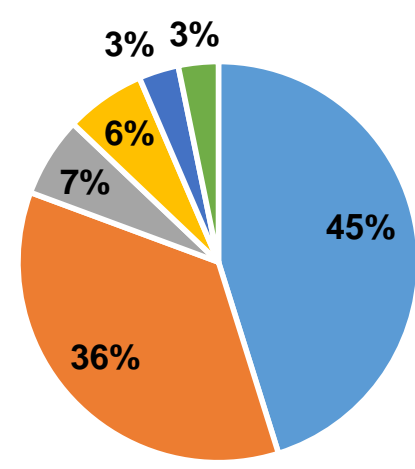

$$
\text { -DIALNET }=\text { SCIELO }=\text { PROQUEST }=\text { REDALYC } \quad \text { EBSCO }=\text { LIBRO }
$$

Fuente: Elaboración Propia 
De la figura se puede observar que la mayoría de información recolectada es de Dialnet con un 45\% (14 artículos), por otra parte, el segundo lugar se encuentra Scielo con un 36\% (11 artículos), de igual modo en el tercer lugar está Proquest y Redalyc con un 7\% (2 artículos), seguidos de Ebsco con un 3\% (1 artículo) y un Libro con 3\%.

Tabla 3

Información recolectada y organizada según título, autor, año, revista científica y base de datos

\begin{tabular}{|c|c|c|c|c|}
\hline Título & Autor & Año & $\begin{array}{l}\text { Revista } \\
\text { Científica }\end{array}$ & $\begin{array}{l}\text { Base de } \\
\text { Datos }\end{array}$ \\
\hline $\begin{array}{l}\text { Revisiones sistemáticas de la } \\
\text { literatura. }\end{array}$ & Beltrán, O. & 2005 & $\begin{array}{l}\text { Revista } \\
\text { Colombiana de } \\
\text { Gastroenterologia }\end{array}$ & Scielo \\
\hline $\begin{array}{l}\text { Estrategia de búsqueda de } \\
\text { información científica aplicada al } \\
\text { reintegro laboral de personas con } \\
\text { discapacidad. } \\
\text { Metodología de la investigación. } \\
\text { México. }\end{array}$ & Galarza, A & 2012 & $\begin{array}{l}\text { Universidad y } \\
\text { Salud, } \\
\text { Red Tercer } \\
\text { Milenio. }\end{array}$ & (Libro) \\
\hline $\begin{array}{l}\text { Beneficios del sector empresarial para } \\
\text { la inclusión laboral de personas con } \\
\text { discapacidad. Un estudio de caso en el } \\
\text { sureste de México. }\end{array}$ & $\begin{array}{l}\text { Hernández, } \\
\text { S., Magaña, } \\
\text { D., y Aquino, } \\
\text { S. }\end{array}$ & 2017 & $\begin{array}{l}\text { Revista } \\
\text { Educación } \\
\text { Inclusiva }\end{array}$ & Dialnet \\
\hline $\begin{array}{l}\text { Rol de la Economía Social y la } \\
\text { Universidad en orden a un } \\
\text { emprendimiento basado en el } \\
\text { conocimiento tecnológico y los } \\
\text { valores. }\end{array}$ & $\begin{array}{l}\text { Julia, J., } \\
\text { Meliá, E., y } \\
\text { Miranda, E. }\end{array}$ & 2020 & CIREC & Dialnet \\
\hline $\begin{array}{l}\text { Modelo de empleo con apoyo para la } \\
\text { inserción laboral de las personas con } \\
\text { discapacidad intelectual leve en } \\
\text { Ecuador. }\end{array}$ & $\begin{array}{l}\text { Lucero, P., y } \\
\text { Romero, R. }\end{array}$ & 2016 & $\begin{array}{l}\text { Revista } \\
\text { Publicando }\end{array}$ & Dialnet \\
\hline $\begin{array}{l}\text { Inclusión sociolaboral de las personas } \\
\text { con discapacidad. Mediación laboral. }\end{array}$ & Manuera, M. & 2015 & $\begin{array}{l}\text { Mediación } \\
\text { laboral. Ciencias } \\
\text { Sociales y de } \\
\text { Comunicacíon }\end{array}$ & Dialnet \\
\hline $\begin{array}{l}\text { Inclusión laboral de personas } \\
\text { catalogadas como discapacitadas: } \\
\text { Algunas reflexiones para un debate } \\
\text { necesario. }\end{array}$ & Mareño, S. & 2015 & $\begin{array}{l}\text { Trabajo } \\
\text { Sociedad }\end{array}$ & Scielo \\
\hline $\begin{array}{l}\text { Inclusión sociolaboral de las personas } \\
\text { con discapacidad en empresas de la } \\
\text { ciudad de Cartagena de Indias. }\end{array}$ & $\begin{array}{l}\text { Marrugo, L., } \\
\text { y Bernal, O. }\end{array}$ & 2017 & Aglala & Dialnet \\
\hline
\end{tabular}




\begin{tabular}{|c|c|c|c|c|}
\hline Título & Autor & Año & $\begin{array}{l}\text { Revista } \\
\text { Científica }\end{array}$ & $\begin{array}{l}\text { Base de } \\
\text { Datos }\end{array}$ \\
\hline $\begin{array}{l}\text { Comunicación interna incluyente: dos } \\
\text { estudios de caso de inclusión laboral } \\
\text { de personas con discapacidad auditiva } \\
\text { en Bogotá. }\end{array}$ & Meléndez, S. & 2016 & $\begin{array}{l}\text { Investigación } \\
\text { Desarrollo }\end{array}$ & Scielo \\
\hline $\begin{array}{l}\text { Avanzando hacia la igualdad de } \\
\text { oportunidades en la inclusión socio- } \\
\text { laboral de las personas con } \\
\text { discapacidad. }\end{array}$ & $\begin{array}{l}\text { Mercado, E., } \\
\text { Aizpurúa, E., } \\
\text { y García, L. }\end{array}$ & 2013 & $\begin{array}{l}\text { Cuaderno de } \\
\text { Trabajo Social }\end{array}$ & Dialnet \\
\hline $\begin{array}{l}\text { Diversidad auditiva: imaginarios } \\
\text { sociales e inclusión laboral: una } \\
\text { aproximación intercultural. }\end{array}$ & Morales, G. & 2015 & Psicogente, & Dialnet \\
\hline $\begin{array}{l}\text { Modelo para la promoción de la } \\
\text { cultura emprendedora como } \\
\text { instrumento de Responsabilidad } \\
\text { Social: caso chileno. }\end{array}$ & $\begin{array}{l}\text { Morales, L., } \\
\text { Hermosilla, } \\
\text { J., y Azua, D. }\end{array}$ & 2019 & Encuentros & Proquest \\
\hline $\begin{array}{l}\text { Revisiones Sistemáticas: definición y } \\
\text { nociones básicas. }\end{array}$ & $\begin{array}{l}\text { Moreno, B., } \\
\text { Muñoz, M., } \\
\text { Cuellar, J., } \\
\text { Domancic, } \\
\begin{array}{l}\text { S., y } \\
\text { Villanueva, J. }\end{array}\end{array}$ & 2018 & $\begin{array}{l}\text { Revista clínica de } \\
\text { periodoncia, } \\
\text { implantología y } \\
\text { rehabilitación }\end{array}$ & Scielo \\
\hline $\begin{array}{l}\text { Valoración ocupacional de las } \\
\text { personas con discapacidad, desde la } \\
\text { perspectiva de las capacidades } \\
\text { humanas. }\end{array}$ & $\begin{array}{l}\text { Moreno, M., } \\
\text { Cortés, E., } \\
\text { Cárdenas, A., } \\
\text { Giraldo, Z., y } \\
\text { Mena, L }\end{array}$ & 2013 & $\begin{array}{l}\text { Revista de Salud } \\
\text { Pública }\end{array}$ & Scielo \\
\hline $\begin{array}{l}\text { Emprendimiento e innovación en } \\
\text { personas con discapacidad en } \\
\text { Colombia: estudio de casos. }\end{array}$ & $\begin{array}{l}\text { Núñez- } \\
\text { Bravo, N., } \\
\text { Sánchez- } \\
\text { Güette, L., } \\
\text { Sotelo- } \\
\text { Berrio, V., } \\
\text { Miranda- } \\
\text { Medina, C., } \\
\text { Mantilla- } \\
\text { Morrón, M., } \\
\text { Galeano- } \\
\text { Muñoz, L., y } \\
\text { Moreno- } \\
\text { Bravo, M. }\end{array}$ & 2019 & $\begin{array}{l}\text { Archivos } \\
\text { Venezolanos de } \\
\text { Farmacologia y } \\
\text { Terapéutica }\end{array}$ & Proquest \\
\hline $\begin{array}{l}\text { Los efectos de las cargas } \\
\text { institucionales en la dicotomía } \\
\text { emprendedora: Oportunidad } \\
\text { necesidad. }\end{array}$ & $\begin{array}{l}\text { Parga, N., y } \\
\text { Silva, M. }\end{array}$ & 2020 & $\begin{array}{l}\text { Contaduría } \quad \text { y } \\
\text { administración }\end{array}$ & Dialnet \\
\hline
\end{tabular}


Emprendimiento como alternativa de inclusión laboral en personas con discapacidad en Latinoamérica: Una revisión sistemática ente los años 2010-2020

\begin{tabular}{|c|c|c|c|c|}
\hline Título & Autor & Año & $\begin{array}{l}\text { Revista } \\
\text { Científica } \\
\end{array}$ & $\begin{array}{l}\text { Base de } \\
\text { Datos }\end{array}$ \\
\hline $\begin{array}{l}\text { Perfil emprendedor en la formación } \\
\text { universitaria venezolana. }\end{array}$ & $\begin{array}{l}\text { Paz, } \\
\text { Salom, } \\
\text { García, J., y } \\
\text { Suarez, } \\
\text { H. }\end{array}$ & 2020 & $\begin{array}{l}\text { Revista de } \\
\text { Ciencias Sociales }\end{array}$ & Dialnet \\
\hline $\begin{array}{l}\text { Beneficios del sector empresarial para } \\
\text { la inclusión laboral de personas con } \\
\text { discapacidad. Un estudio de caso en el } \\
\text { sureste de México. }\end{array}$ & $\begin{array}{l}\text { Pérez, S., } \\
\text { Magaña, D., } \\
\text { y Aquino, S. }\end{array}$ & 2017 & $\begin{array}{l}\text { Revista } \\
\text { Educación } \\
\text { Inclusiva }\end{array}$ & Dialnet \\
\hline $\begin{array}{l}\text { Mejores prácticas empresariales de } \\
\text { responsabilidad social en la inclusión } \\
\text { de personas con discapacidad. } \\
\text { Estudio de caso en empresas de } \\
\text { Ambato, Ecuador. }\end{array}$ & $\begin{array}{l}\text { Pico, P., y } \\
\text { Torres, S. }\end{array}$ & 2017 & $\begin{array}{l}\text { Revista de } \\
\text { Ciencias de la } \\
\text { Administración y } \\
\text { Economía }\end{array}$ & Dialnet \\
\hline $\begin{array}{l}\text { Inclusión social y laboral de las } \\
\text { personas con discapacidad desde la } \\
\text { articulación socio-sanitaria en el Perú. }\end{array}$ & $\begin{array}{l}\text { Quiroz, L., } \\
\text { Cordero, J., y } \\
\text { Giraldo, E. }\end{array}$ & 2017 & $\begin{array}{l}\text { CASUS: Revista } \\
\text { de Investigación } \\
\text { y Casos en Salud }\end{array}$ & Dialnet \\
\hline $\begin{array}{l}\text { por empresas privadas inclusivas de } \\
\text { Asunción, en el proceso de inclusión } \\
\text { laboral de Personas con Discapacidad } \\
\text { (PcD), Año } 2013 \text {. }\end{array}$ & $\begin{array}{l}\text { Ramírez, C., } \\
\text { y Ramírez, D. }\end{array}$ & 2015 & $\begin{array}{l}\text { Población } \\
\text { Desarrollo }\end{array}$ & Dialnet \\
\hline $\begin{array}{l}\text { Empleabilidad de personas con } \\
\text { discapacidad desde el marco rector de } \\
\text { SENADIS, Paraguay. }\end{array}$ & Reyes, A. & 2015 & $\begin{array}{l}\text { Revista } \\
\text { Internacional de } \\
\text { Investigación en } \\
\text { Ciencias Sociales }\end{array}$ & Scielo \\
\hline $\begin{array}{l}\text { Inserción laboral y desarrollo } \\
\text { profesional de las personas con } \\
\text { discapacidades en Empresas } \\
\text { Turísticas de la ciudad de Loja. }\end{array}$ & $\begin{array}{l}\text { Sánchez, J., } \\
\text { Román, R., } \\
\text { Torres, A., y } \\
\text { Loarte, M. }\end{array}$ & 2019 & $\begin{array}{l}\text { Revista } \\
\text { Universidad } \\
\text { Sociedad }\end{array}$ & Scielo \\
\hline $\begin{array}{l}\text { Inclusión laboral de personas } \\
\text { catalogadas como discapacitadas: } \\
\text { Algunas reflexiones para un debate } \\
\text { necesario. }\end{array}$ & $\begin{array}{l}\text { Sempertegui, } \\
\text { M }\end{array}$ & 2015 & $\begin{array}{l}\text { Trabajo } \\
\text { sociedad }\end{array}$ & Scielo \\
\hline Implicaciones culturales $\quad y$ & & & & \\
\hline $\begin{array}{l}\text { socioeconómicas de la mujer como } \\
\text { partícipe en el desarrollo y } \\
\text { fortalecimiento de los sectores } \\
\text { económicos: un análisis comparativo } \\
\text { México-China. }\end{array}$ & Tello, J. & 2016 & $\begin{array}{lr}\text { Revista en } & \text { línea } \\
\text { Mundo } & \text { Asia } \\
\text { Pacifico } & \end{array}$ & Ebsco \\
\hline $\begin{array}{l}\text { Análisis crítico sobre el uso del SROI } \\
\text { en la evaluación del impacto social en } \\
\text { iniciativas de emprendimiento social: } \\
\text { caso México. }\end{array}$ & $\begin{array}{l}\text { Valdés, F., y } \\
\text { Saavedra, M. }\end{array}$ & 2019 & Ad-minister & Scielo \\
\hline
\end{tabular}




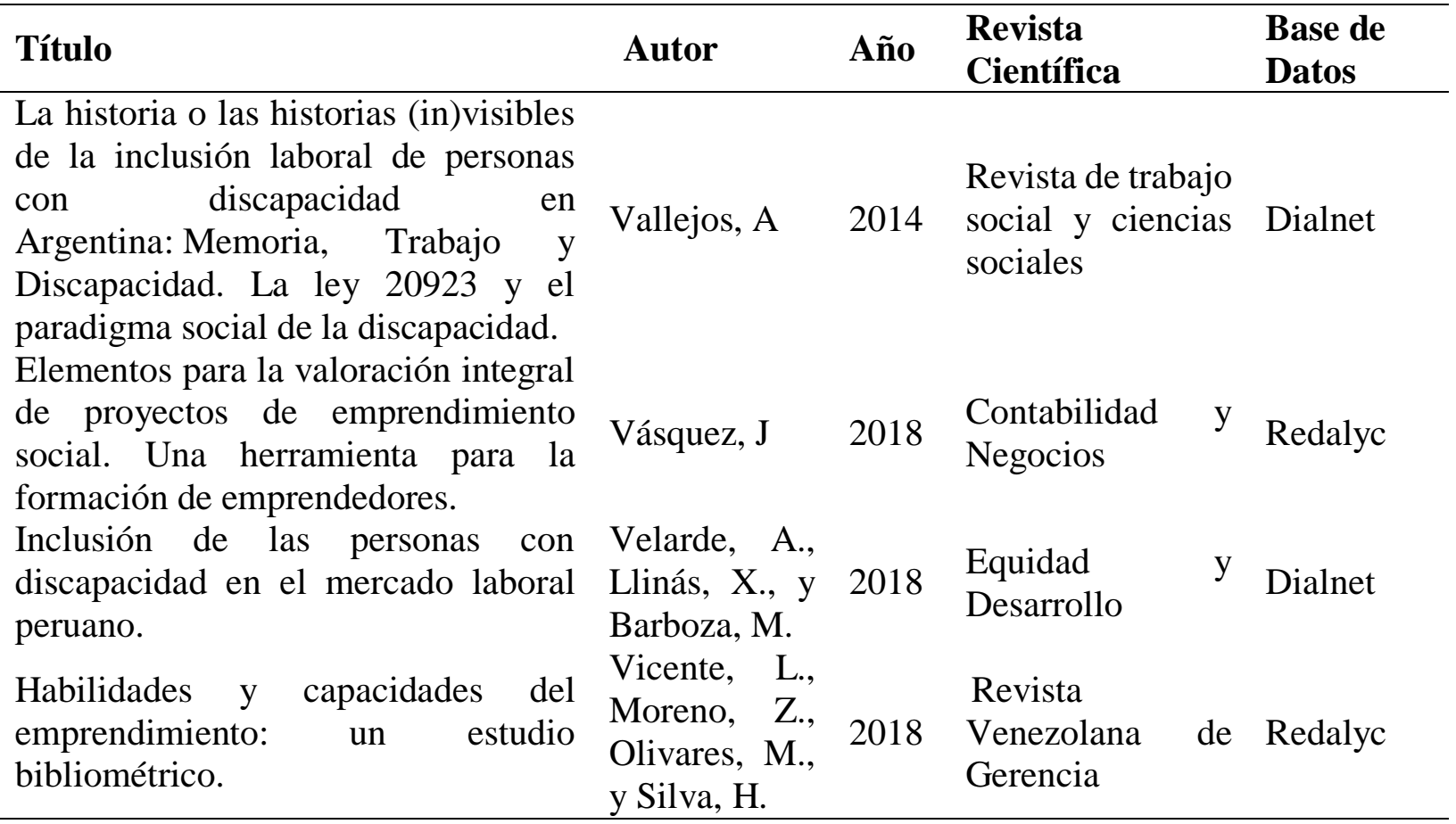

En la presente tabla podemos apreciar la información recolectada de los 32 artículos, que permitieron el desarrollo de la presente revisión sistemática.

\section{Discapacidad}

En general, la discapacidad es un término que presenta diversas definiciones sujetas a diferentes criterios o paradigmas (Núñez-Bravo et al., 2019). En la literatura revisada se hallaron diferentes acepciones sobre discapacidad que pueden ser agrupadas en 4 categorías. La primera categoría se refiere a la estadística de población con algún tipo de discapacidad que sería el $15 \%$ de la Población, con poca oportunidad de mejorar su calidad de vida (Galarza, 2020).

Una segunda categoría asume el ámbito normativo en el aspecto laboral, existen política Internacionales y Nacionales que buscan amparar a la Población con Discapacidad (Quiroz et al., 2017); Así como La Organización Internacional del Trabajo en el año 2015, busca la igualdad de condiciones y remuneraciones en el ámbito laboral (Sánchez et al., 2019), es así que la Convención Internacional de los Derechos de las Personas con Discapacidad impulsa la inclusión laboral bajo una perspectiva de oportunidad y accesibilidad laboral (Moreno et al., 2013).

La tercera categoría está adscrita a la función social, donde surge un "Paradigma Social de la Discapacidad", poniendo énfasis en las barreras que hay en la interacción de las personas y su entorno, más que en su condición personal, ello limita su participación en la vida social (Vallejos, 2014), sumado a ello la limitación para su empleabilidad e inserción laboral, que genera una gran problemática social (Sempertegui, 2015).

Una cuarta categoría relacionada a la oportunidad laboral, la población con discapacidad en el mundo enfrenta muchos retos buscando cubrir sus necesidades básicas en salud, vivienda, 
Emprendimiento como alternativa de inclusión laboral en personas con discapacidad en Latinoamérica: Una revisión sistemática ente los años 2010-2020

educación y empleo sin discriminación (Meléndez, 2016); aun así, dicha población tiene poca participación en el ámbito laboral (Pico y Torres, 2017), para ello es necesario el compromiso y la sensibilización de las autoridades, empresas privadas, organizaciones civiles y la misma sociedad para lograr una inclusión social de las Personas con Discapacidad (Hernández et al., 2017), un aspecto importante a tener en cuenta es que la particularidad de la discapacidad puede desempeñar habilidades que mejoren su desempeño laboral en busca de un entorno más accesible (Morales, 2015).

\section{Inclusión Laboral}

La revisión de la literatura comprende como el concepto de inclusión laboral toma en cuenta a la inserción total e incondicional de las personas en un ámbito laboral (Meléndez, 2016), permitiendo su realización personal y social (Albarrán-Lozano y Alonso-González, 2010). Igualmente, la inclusión laboral permite la construcción de una independencia económica que permita lograr una vida satisfactoria y plena (Calderón-Milán et al., 2020), permitiendo incluso una participación recurrente en actividades cotidianas que mejoren su calidad de vida (Akyurek et al., 2020)

Existen modelos que deben ser adaptados a la situación actual (Reyes, 2015); y se debe desarrollar un modelo de gestión inclusiva que cumpla los requerimientos legales y sociales para las personas con discapacidad (Lucero y Romero, 2016; Alemán, 2018). Aún existe la desigualdad en la esfera productiva, aunque se tengan normas legales que amparen a dicha población (Mercado et al., 2013).

Es preocupante saber que la población con discapacidad aún no son un grupo de interés en las organizaciones (Marrugo y Bernal, 2017). Creándose fallas en torno a la inclusión laboral de dicha población (Pico y Torres, 2017); otro tema importante es buscar la estabilidad laboral de la población con discapacidad reconociendo sus derechos (Manuera, 2015).

Es necesario trabajar con los responsables de las empresas privadas, motivando la inclusión de las personas con discapacidad (Ramírez y Ramírez, 2015); Para ello, el estado debe contar con mecanismos de control adecuados (Velarde et al., 2018).

\section{Emprendimiento}

En el análisis de la literatura da cuenta que, en la última década, el emprendimiento se ha convertido en una estrategia social que desarrolla habilidades para realizar proyectos empresariales (Vicente et al., 2018). El emprendimiento surge como alternativa para mejorar la economía de un país (Diez Farhat et al., 2021) pudiendo verse vinculado a la informalidad (Vega y Bermúdez, 2019), es realizado por personas con diferentes motivaciones que varían con el tiempo y las circunstancias (Tovar et al., 2018; Ynzunza y Izar, 2021). Además, se comprende como un actor fundamental en la realización de una verdadera inclusión laboral tanto para las personas con discapacidad (Pico y Torres, 2017), como para mujeres que emprenden por necesidad (Hermoso, 2016). En la actualidad un emprendimiento innovador busca una sociedad más justa solidaria y sostenible (Julia et al., 2020). Aunque se manifiesta que el emprendimiento empresarial busca metas de innovación, crecimiento económico y desarrollo social (Tello, 2016). 
El emprendimiento surge también como un enfoque social a partir de un modelo de negocio inclusivo (Vásquez, 2018), formando parte de la solución a una problemática social (Valdés y Saavedra, 2019). En concreto, el emprendimiento supone una forma de incluir al mercado laboral a un grupo que, generalmente, suele discriminar a individuos que sufren algún tipo de discapacidad (Casado y Casaú, 2019)

Un emprendedor utiliza un ecosistema emprendedor que une condiciones económicas y sociales (Parga y Silva, 2020); el emprendedor se convierte en un agente activo que aporta ideas creativas e innovadoras (Paz et al., 2020). La persona con discapacidad tiene muchas motivaciones para emprender (Núñez et al., 2019). A pesar de ello, es necesario recalcar que los individuos con condiciones adversas pueden sufrir discriminación al momento de solicitar servicios financieros que permitan solventar sus proyectos (Sarker, 2020)

\section{Conclusiones}

La presente revisión sistemática de la literatura científica, sobre Emprendimiento e Inclusión laboral en personas con discapacidad durante los últimos 10 años, se encontró analizando un total de 41 artículos y 1 libro publicados en los años 2010 - 2020, los cuales tenían una relación con el tema de "Emprendimiento e inclusión laboral", brindando conceptos y sustentos normativos que amparan ambos términos.

Se pudo encontrar que no hay muchos estudios referentes al emprendimiento como alternativa de inclusión laboral a en personas con discapacidad, siendo una problemática social que tiene muchas falencias debido a que este grupo de población busca satisfacer sus necesidades de crecimiento y desarrollo.

El emprendimiento surge como una alternativa de respuesta amparada en normas y leyes internacionales que, aunque tienen ciertas falencias, su impulso social lo lleva a enfocarse en la inclusión laboral de las personas con discapacidad, desde un emprendimiento social e inclusivo sin discriminación. Los autores buscan un mismo objetivo, con sustentos más sociales e inclusivos

La población con discapacidad tiene poca oportunidad laboral, aunque existen normas y leyes que amparen la inclusión laboral con equidad y respeto, no se cumple para beneficio de esta población. Es necesario sensibilizar a las autoridades y la sociedad para comprometerse en brindarle oportunidades laborales a las personas con discapacidad que necesitan cubrir sus necesidades, romper paradigmas y barreras de discriminación.

El emprendimiento y la inclusión laboral surgen como alternativas de solución viéndose desde un aspecto económico y social. Surge un antagonismo visible entre el avance en normas y leyes para beneficio de las personas con discapacidad y la falta de sensibilidad y compromiso humano, un aspecto social hablado, pero no practicado. Es así que se recomienda la concientización de la sociedad sobre la inclusión laboral y la fomentación de normas de contratación a personas con discapacidad en las empresas y los controles respectivos para su estabilidad laboral. 
A pesar de las limitaciones expuestas, se debe tomar en cuenta y valorar los hallazgos de este estudio como una base teórica que impulsa a generarse nuevas preguntas con respecto al emprendimiento e inclusión laboral, partiendo del conocimiento sobre los medios, procesos, alternativas de oportunidad laboral, descritas y categorizadas en este estudio. Se sugiere realizar una investigación empírica, cuantitativa, multivariada, que analice causas y efectos en temas relacionados a la inserción laboral de personas con discapacidad.

\section{Referencias bibliográficas}

Akyurek, G., Bumin, G., \& Crowe, T. K. (2020). The factors associated with community participation: Employment and education of people with disabilities in Turkey. Scandinavian Journal of Occupational Therapy, 27(1), 28-38. https://doi.org/10.1080/11038128.2019.1587502

Albarrán-Lozano, I., y Alonso-González, P. (2010). Participación en el mercado laboral español de las personas con discapacidad y en situación de dependencia. Papeles de Población, 16(64), 217-256. http://www.scielo.org.mx/scielo.php?script=sci_arttext\&pid=S140574252010000200009

Alemán, J. P. (2018). State duties regarding the inclusion in the labor market of persons with disabilities. Revista Latinoamericana de Derecho Social, 1(26), 155-216. https://doi.org/10.22201/iij.24487899e.2018.26.11863

Beltrán, O. (2005). Revisiones sistemáticas de la literatura. Revista Colombiana de Gastroenterologia, 20(1). 60-69. https://www.redalyc.org/articulo.oa?id=337729264009

Calderón-Milán, M. J., Calderón-Milán, B., y Barba-Sánchez, V. (2020). Inclusión laboral de personas con discapacidades: ¿Qué papel desempeñan las entidades de la economía social y solidaria? Sustainability (Switzerland), 12(3). https://doi.org/10.3390/su12031079

Casado, A., \& Casaú, P. (2019). Personal self-knowledge, a key factor for entrepreneurship in people with disabilities. Journal of Entrepreneurship Education, 22(2). https://www.researchgate.net/publication/335777114_Personal-self-knowledge-a-keyfactor-1528-2651-22-S2-433

Diez Farhat, S., Vargas Valdiviezo, M. A., y Acosta Ramírez, N. (2021). Análisis estructural de los factores que inciden en el emprendimiento. Revista Venezolana de Gerencia, 26(93), 334-348. https://doi.org/10.52080/rvg93.23

Galarza, A. (2020). Estrategia de búsqueda de información científica aplicada al reintegro laboral de personas con discapacidad. Universidad y Salud, 22(2), 186-197. https://doi.org/10.22267/rus.202202.190

Gomez, S. (2012). Metodología de la investigación. Red Tercer Milenio.

Hermoso, Ú. N. (2016). Análisis del emprendimiento femenino atendiendo a la influencia del rol de la mujer en el acceso al mercado laboral y a la educación superior. Opción, 32(10), 394411. https://dialnet.unirioja.es/servlet/articulo?codigo=5875173

Hernández, S., Magaña, D., y Aquino, S. (2017). Beneficios del sector empresarial para la inclusión laboral de personas con discapacidad. Un estudio de caso en el sureste de México. Revista de Educación Inclusiva, https://dialnet.unirioja.es/servlet/articulo?codigo $=7532204$ 
Julia, J., Meliá, E., y Miranda, E. (2020). Rol de la Economía Social y la Universidad en orden a un emprendimiento basado en el conocimiento tecnológico y los valores. CIRIEC - Espana, 98, 31-57. https://doi.org/10.7203/CIRIEC-E.98.15905

Lucero, P., y Romero, R. (2016). Modelo de empleo con apoyo para la inserción laboral de las personas con discapacidad intelectual leve en Ecuador. Revista Publicando, 3(7), 57-68. https://dialnet.unirioja.es/servlet/articulo?codigo $=5833451$

Manuera, M. (2015). Inclusión sociolaboral de las personas con discapacidad. Mediación laboral. Ciencias Sociales y de Comunicacíon, 14, 161-179. https://doi.org/10.5209/rev_MESO.2015.n14.51564

Mareño, S. (2015). Inclusión laboral de personas catalogadas como discapacitadas: Algunas reflexiones para un debate necesario. Trabajo y sociedad, 25, 405-442. http://www.scielo.org.ar/pdf/tys/n25/n25a23.pdf

Marrugo, L., y Bernal, O. (2017). Inclusión sociolaboral de las personas con discapacidad en empresas de la ciudad de Cartagena de Indias. Aglala, 8(1), 282-315. https://doi.org/10.22519/22157360.1036

Meléndez, S. (2016). Comunicación interna incluyente: dos estudios de caso de inclusión laboral de personas con discapacidad auditiva en Bogotá. Investigación y Desarrollo, 24(1), 2652. https://doi.org/10.14482/indes.24.1.8683

Mercado, E., Aizpurúa, E., y García, L. (2013). Avanzando hacia la igualdad de oportunidades en la inclusión socio-laboral de las personas con discapacidad. Cuaderno de Trabajo Social, 26(1), 95-104. https://dialnet.unirioja.es/servlet/articulo?codigo=4376524

Morales, G. (2015). Diversidad auditiva: imaginarios sociales e inclusión laboral: una aproximación intercultural. $\quad$ Psicogente, $\quad$ 18(34), 364-371. https://dialnet.unirioja.es/servlet/articulo?codigo=6113769

Morales, L., Hermosilla, J., y Azua, D. (2019). Modelo para la promoción de la cultura emprendedora como instrumento de Responsabilidad Social: caso chileno. Encuentros, 17(2), 165-177. https://doi.org/10.15665/encuent.v17i02.1796

Moreno, B., Muñoz, M., Cuellar, J., Domancic, S., y Villanueva, J. (2018). Revisiones Sistemáticas: definición y nociones básicas. Revista clínica de periodoncia, implantología y rehabilitación oral, 11(3), 184 - 186. http://doi.org/10.4067/S0719-01072018000300184

Moreno, M., Cortés, E., Cárdenas, A., Giraldo, Z., y Mena, L. (2013). Valoración ocupacional de las personas con discapacidad, desde la perspectiva de las capacidades humanas. Revista de Salud Pública, 15(5), 764-777. https://scielosp.org/pdf/rsap/2013.v15n5/764-777

Núñez-Bravo, N., Sánchez-Güette, L., Sotelo-Berrio, V., Miranda-Medina, C., Mantilla-Morrón, M., Galeano-Muñoz, L., y Moreno-Bravo, M. (2019). Emprendimiento e innovación en personas con discapacidad en Colombia: estudio de casos. Archivos Venezolanos de Farmacologia y Terapéutica, 38(2), 159-165. https://hdl.handle.net/20.500.12442/4158

Parga, N., y Silva, M. (2020). Los efectos de las cargas institucionales en la dicotomía emprendedora: Oportunidad o necesidad. Contaduría y administración, 65(2). https://dialnet.unirioja.es/servlet/articulo?codigo=7357963

Paz, A., Salom, J., García, J., y Suarez, H. (2020). Perfil emprendedor en la formación universitaria venezolana. Revista de Ciencias Sociales, 26(1), 161-174. https://dialnet.unirioja.es/servlet/articulo?codigo=7384412

Pérez, S., Magaña, D., y Aquino, S. (2017). Beneficios del sector empresarial para la inclusión laboral de personas con discapacidad. Un estudio de caso en el sureste de México. Revista de Educación Inclusiva, 1(1). https://dialnet.unirioja.es/servlet/articulo?codigo=7532204 
Emprendimiento como alternativa de inclusión laboral en personas con discapacidad en Latinoamérica: Una revisión sistemática ente los años 2010-2020

Pico, P., y Torres, S. (2017). Mejores prácticas empresariales de responsabilidad social en la inclusión de personas con discapacidad. Estudio de caso en empresas de Ambato, Ecuador. RETOS. Revista de Ciencias de la Administración y Economía, 7(14), 189-200. https://doi.org/10.17163/ret.n14.2017.10.

Quiroz, L., Cordero, J., y Giraldo, E. (2017). Inclusión social y laboral de las personas con discapacidad desde la articulación socio-sanitaria en el Perú. CASUS: Revista de $\begin{array}{llllll}\text { Investigación } & y & \text { Casos } & \text { en } & \text { Salud, } & \text { 179-184. }\end{array}$ https://dialnet.unirioja.es/servlet/articulo?codigo $=6258769$

Ramírez, C., y Ramírez, D. (2015). Estrategias e indicadores utilizados por empresas privadas inclusivas de Asunción, en el proceso de inclusión laboral de Personas con Discapacidad (PcD), Año 2013. Población y Desarrollo, 40, 8-25. https://dialnet.unirioja.es/servlet/articulo?codigo $=5654225$

Reyes, A. (2015). Empleabilidad de personas con discapacidad desde el marco rector de SENADIS, Paraguay. Revista Internacional de Investigación en Ciencias Sociales, 11(22), 209-222. https://doi.org/10.18004/riics.2015.diciembre.209-222

Sánchez , J., Román, R., Torres, A., y Loarte, M. (2019). Inserción laboral y desarrollo profesional de las personas con discapacidades en Empresas Turísticas de la ciudad de Loja. Revista $\begin{array}{lllll}\text { Universidad } & y & \text { Sociedad, 201-205. }\end{array}$ http://scielo.sld.cu/scielo.php?script=sci_arttext\&pid=S2218$36202019000500201 \& \operatorname{lng}=$ es\&nrm=iso

Sarker, D. (2020). Discrimination against people with disabilities in accessing microfinance. Alter, 14(4), 318-328. https://doi.org/10.1016/j.alter.2020.06.005

Sempertegui, M. (2015). Inclusión laboral de personas catalogadas como discapacitadas: Algunas reflexiones para un debate necesario. Trabajo y sociedad, 25, 405-442. http://www.scielo.org.ar/pdf/tys/n25/n25a23.pdf

Tello, J. (2016). Implicaciones culturales y socioeconómicas de la mujer como partícipe en el desarrollo y fortalecimiento de los sectores económicos: un análisis comparativo MéxicoChina. Revista en línea Mundo Asia Pacifico, 5(9), 24-43. https://doi.org/10.17230/map.v5.i9.02

Tovar, L., Balanta, S., Ordoñez, J., y Serna, W. (2018). Factores asociados al emprendimiento por oportunidad de colombianos retornados del exterior. Migraciones, 45, 119-142. https://doi.org/mig.i45.y2018.005

Valdés, F., y Saavedra, M. (2019). Análisis crítico sobre el uso del SROI en la evaluación del impacto social en iniciativas de emprendimiento social: caso México. Ad-minister, 35, 5376. https://doi.org/10.17230/Ad-minister.35.2

Vallejos, A. (2014). La historia o las historias (in)visibles de la inclusión laboral de personas con discapacidad en Argentina: Memoria, Trabajo y Discapacidad. La ley 20923 y el paradigma social de la discapacidad. Revista de trabajo social y ciencias sociales, 74, 3277585. https://dialnet.unirioja.es/servlet/articulo?codigo=5170611

Vásquez, J. (2018). Elementos para la valoración integral de proyectos de emprendimiento social. Una herramienta para la formación de emprendedores. Contabilidad y Negocios, 13(26), 129-140. https://doi.org/10.18800/contabilidad.201802.008

Vega, C., y Bermúdez, H. F. (2019). Informalidad, emprendimiento y empoderamiento femenino. Economía popular y paradojas de la venta directa en el sur de Quito (Ecuador). Revista de Antropología Social, 28(2), 345-370. https://doi.org/10.5209/raso.65618

Esta obra se comparte bajo la licencia Creative Common Atribución-No Comercial 4.0 International (CC BY-NC 4.0) 
Velarde, A., Llinás, X., y Barboza, M. (2018). Inclusión de las personas con discapacidad en el mercado laboral peruano. Equidad y Desarrollo, 32, 57-58. https://dialnet.unirioja.es/servlet/articulo?codigo $=6771628$

Vicente, L., Moreno, Z., Olivares, M., y Silva, H. (2018). Habilidades y capacidades del emprendimiento: un estudio bibliométrico. Revista Venezolana de Gerencia, 23(81), 12511256. https://www.redalyc.org/jatsRepo/290/29055767013/html/index.html

Ynzunza, C. B., \& Izar, J. M. (2021). The impact of motivations, competencies and success factors for entrepreneurship on business performance. An analysis in MSMES in Queretaro State, México. Contaduría y Administración, 66(1), 1-26. https://doi.org/10.22201/fca.24488410e.2021.2327 